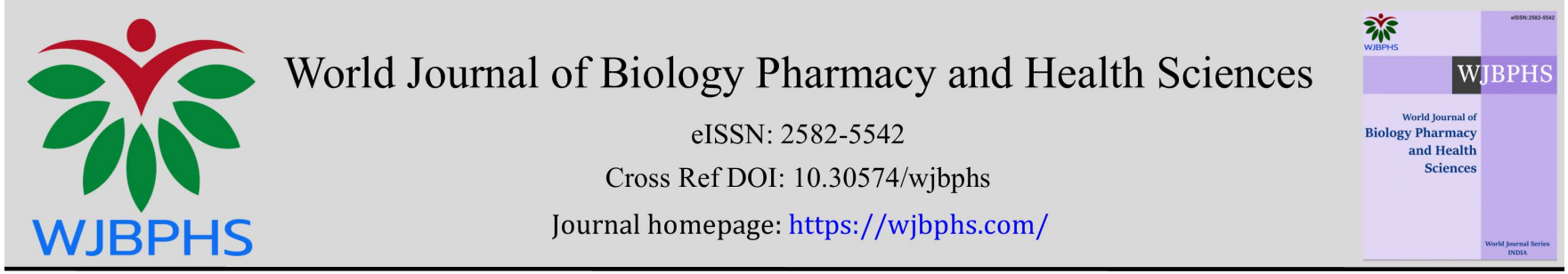

(CASE REPORT)

\title{
Olfactory neuroblastoma: case report and focus on cancerogenesis
}

\author{
Stefania Erra ${ }^{1}$ and Ennio Nano B D ${ }^{2, *}$ \\ ${ }^{1}$ Surgical Pathology Department, Santo Spirito Hospital, Viale Giolitti 2, 15033, Casale Monferrato (ITALY) \\ 2 University of Eastern Piedmont Amedeo Avogadro, Viale Teresa Michel 11, Alessandria (ITALY).
}

World Journal of Biology Pharmacy and Health Sciences, 2021, 06(03), 035-039

Publication history: Received on 25 April 2021; revised on 14 June 2021; accepted on 17 June 2021

Article DOI: https://doi.org/10.30574/wjbphs.2021.6.3.0060

\begin{abstract}
Background: Neuroendocrine neoplasms (NENs) of the nasal cavity, paranasal sinuses and nasopharynx account for a wide spectrum of histotypes. They can range from indolent form to highly aggressive tumors. Olfactory neuroblastoma, like most sino-nasal NENs, represents a rare neoplasm that prompts diagnostic pitfalls. From a morphological perspective, olfactory neuroblastoma can mimic many nasal neoplasms but a proper recognition is mandatory for its aggressive behaviour.
\end{abstract}

Case presentation: A case of olfactory neuroblastoma in a 85 years old woman is reported. The neoplasm has been surgical removed from the nasal cavity with a clinical and radiographic diagnosis of nasal polyp. Correct diagnostic definition has needed a complete histological and immunohistochemical characterization of the tumoral tissue in surgical pathology laboratory.

Conclusion: The correct diagnosis of olfactory neuroblastoma directs the clinical management that is unique for this neoplasm in comparison to other nasal ones. Bicranial-facial surgery or a trephination procedure represent the most common approach for the treatment of this malignancy. Their success determine disease prognosis.

Keywords: Olfactory neuroblastoma; Sinonasal-tract; Neuroendocrine neoplasm; Immunohistochemistry; Synaptofisin

\section{Background}

Olfactory neuroblastomas (ONBs) constitute the neuroectodermal class of neuroendocrine sino-nasal neoplasms. This class differentiates from the sino-nasal neuroendocrine carcinomas group that follows the lung neuroendocrine neoplasm classification. Cases of sino-nasal neoplasms composed of neuroendocrine and non-neuroendocrine component have been documented in literature. They don't fit the classification of nasal neuroendocrine neoplasm and actually are not included in WHO classification [1]. ONBs are rare malignant neoplasms that account for 2\% of all sinonasal tract tumors. Incidence of ONBs accounts approximately 0.4 per million population [2]. ONBs equally affect individuals of both sexes and occur in an age interval ranging from 2 to 90 years. The incidence age distribution of ONBs shows a bimodal morphology with two peak of incidence respectively in the 5 th and 6 th decades.

Clinical manifestations of ONBs include common symptoms: 70\% of the patients present unilateral nasal obstruction, $50 \%$ of cases have epistaxis. Less common signs of ONBs include nonspecific symptoms such as pain, headaches, excessive lacrimation, rhinorrea, anosmia and visual disturbances. Reports of isolated cases of vasopressin secreting ONBs associated with hypertension and hyponatremia symptoms are described in literature. Lack of patognomonic symptoms in addition to an initial tumor slow growth can delay the diagnosis of ONBs[2].Clinical suspect of ONB is

\footnotetext{
* Corresponding author: Stefania Erra; Phone number: +390142434894; Email:stefania.erra@virgilio.it Surgical Pathology Department, Santo Spirito Hospital, Viale Giolitti 2, 15033, Casale Monferrato Italy. 
based on characteristic imaging features of the lesion though this observations are related to the size of the tumor, its location and time elapsed from symptoms onset. Imaging finding of ONBs include a "dumbell-shaped" mass arising from and extending across the cribriform plate causing bone erosion, an aspect usually highlighted by CT scan analysis. Magnetic Resonance (MRI) imaging is better suited for ONB staging. MRI is effective in delineating sinonasal, intraorbital or intracerebral tumor exstension, while CT scan is applied to assess erosion of lamina papyracea and cribriform plate.

For its unique biological behavior, ONBs are staged according to alternative staging systems than TNM, although this one could be potentially applied for paranasal sinus tumors staging [3]. Among the staging system proposed to classify ONBs, the most widely applied is the Kadish System, which classifies local spreading neoplasm. It differentiates ONBs based on: involvement of the nasal cavity only; extension into the paranasal sinuses; extension outside the paranasal sinuses [4]. Pathological gross inspection of ONB typically reveals a unilateral polypoid lesion with glistening, soft, redgrey features. The mass usually present with an intact mucosa and its cut-surface appears hypervascular with a greytan to pink-red color [2]. Tumor dimensions can range from $1 \mathrm{~cm}$ to large masses infiltrating adjacent paranasal sinuses, orbits or the cranial vault. From the histological perspective, ONBs are constituted from uniform lobules of small round blue cells surrounded by a neurofibrillary and edematous stroma [5]. Fibrovascular septa support cellular nests. Tumor cells are larger than lymphocytes with a high nuclear to cytoplasmatic ratio; nuclei are small, uniform and hyperchromatic characterized by a salt and pepper chromatin distribution; nucleoli are little and few detectable [2]. Rare patterns of Homer-Wright pseudorosettes and Flexner-Wintersteiner rosettes can be observed [5]. On histology viewpoint, ONBs are classified in four grades based on Hyam grading system [2], based on cell maturation assigning a score to the subsequent key tumor features: mitotic activity, nuclear pleomorphism, rosette formation, necrosis, histologic architecture and presence of the fibrillary stroma [5] [6].

Majority of the ONBs falls into Grade I Hyam class that comprises most differentiated tumors. It is represented from little and uniform cells, with small nuclei and homogenously dispersed chromatin, a syncitial arrangement with cytoplasmatic neurofibrillary exstensions. Stroma is fibrovascular and necrosis and mitotic activity are absent. On the other hand, grade IV ONBs are composed of anaplastic cells characterized by anisocariosis and presenting eosinophilic macronucleoli. Necrosis and atypical mitotic figures are common features of grade IV neoplasms, as the loss of neurofibrillary stroma and calcifications. Immunohistochemical markers confirming ONB diagnosis are: S-100 protein, NFP (neurofibrillary protein), CD56, sinaptophysin, chromogranin and a high proliferative index of 10-50\% established with Ki-67. Negative markers are desmin, myogenin, CD99 and the most of lymphocytic CD markers [2]. Therapeutic approach to ONBs is characterized by multimodality treatment except for localized and indolent tumors that benefit only from surgery [7]. Surgical eradication usually requires a trephination; in some cases radical excision of the neoplasm can be achieved with an endoscopic resection. To better patient outcome, surgery typically is followed by a course of radiotherapy. Chemotherapy usually has a palliative intention and it's adopted in cases of advanced or disseminated disease [2]. Lee D Cramer et al. reviewed neo-adjuvant role of chemotherapy in cases with a primary localization to the nasal cavity, revealing its inefficacy in improving overall and disease-specific survival [8].

\section{Case Presentation}

An 85 years old woman came to our Institution after concerning symptoms of persistent nasal pain, rhinorrea and epistaxis. Endoscopic examination of sino-nasal tract revealed obstruction caused by an ovoid polypoid mass that was excited. Mass dimensions measured 3x1 cm. Microscopic features of the lesion showed a solid and highly vascularized proliferation of small to medium size round cells organized in pseudo cystic spaces and Flexner- Wintersteiner rosettes (figure 1). Necrosis wasn't detected but atypical mitotic figures were identified. Ki67 immunohistochemical marker scored a $10 \%$ proliferation index. Immunophenotype of neoplastic cells showed positive staining for: synaptophysin; CD56; calretinin; INI1; CK8/18 focally; S100 in sustentacular cells (figures 2,3). Negative neoplastic staining included: TTF1; CEA; actin smooth muscle antigen. Immunophenotype of neoplastic cells confirmed the neuroectodermic origin of the lesion. Morphological aspects of the neoplasm were congruent with a Hyams Grade III neuroblastoma diagnosis. 


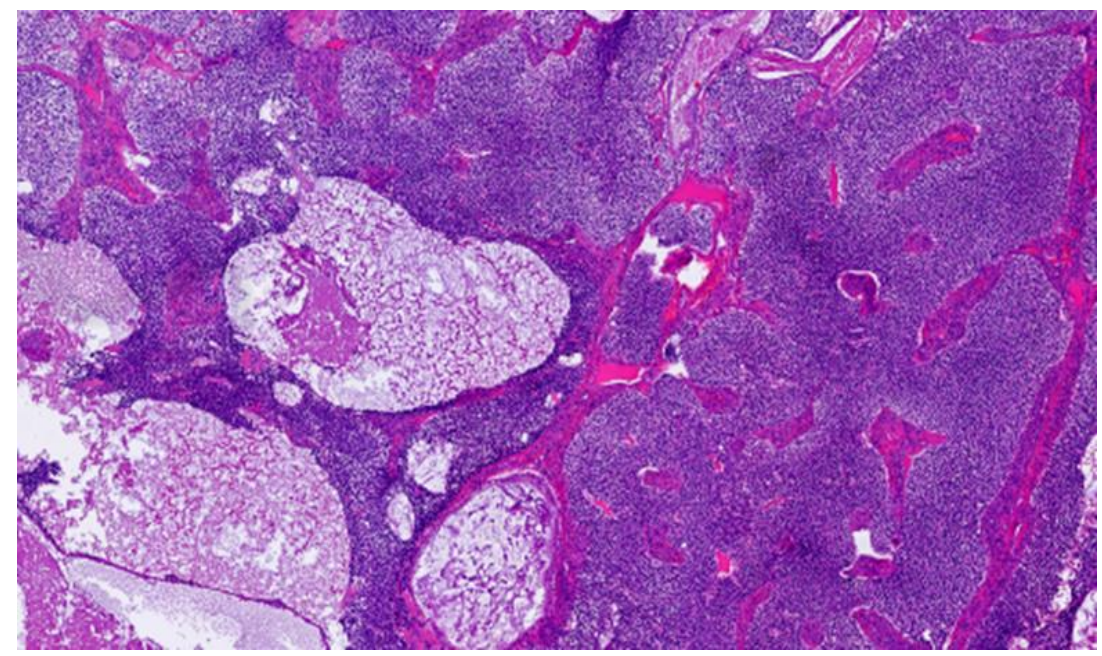

Figure 1 Low magnification of blue small round cells characterized by a polypoid proliferation and organized to delimitate pseudo cystic spaces (on the left)

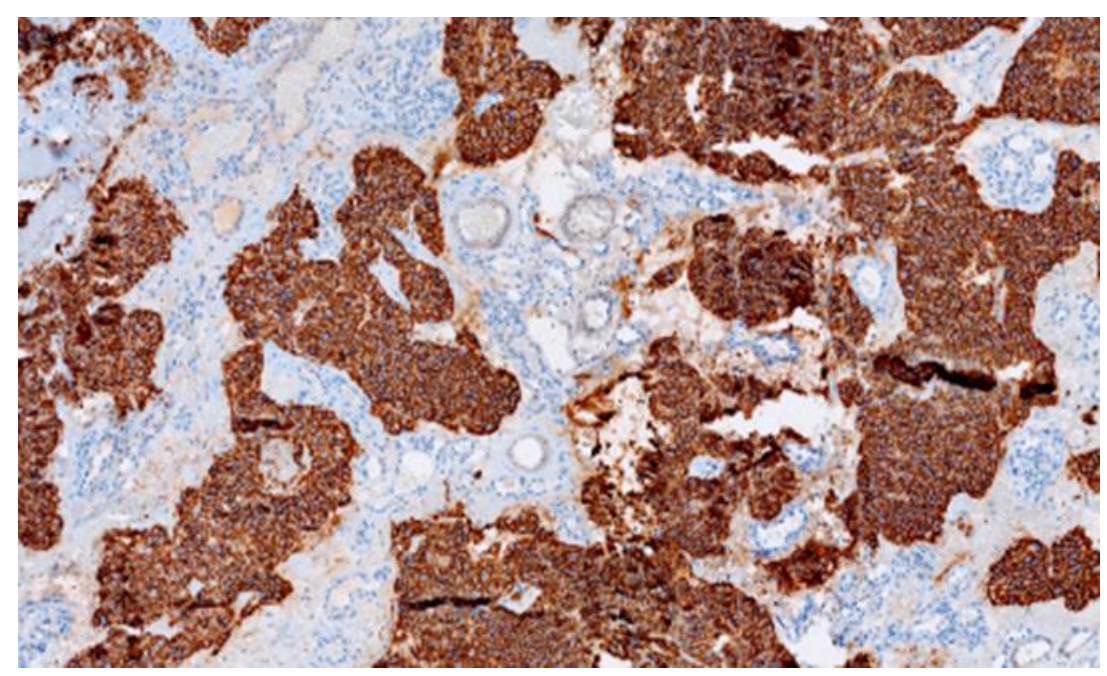

Figure 2 Immunohistochemical staining for CD56 showing positivity in the neoplastic component of the tumor

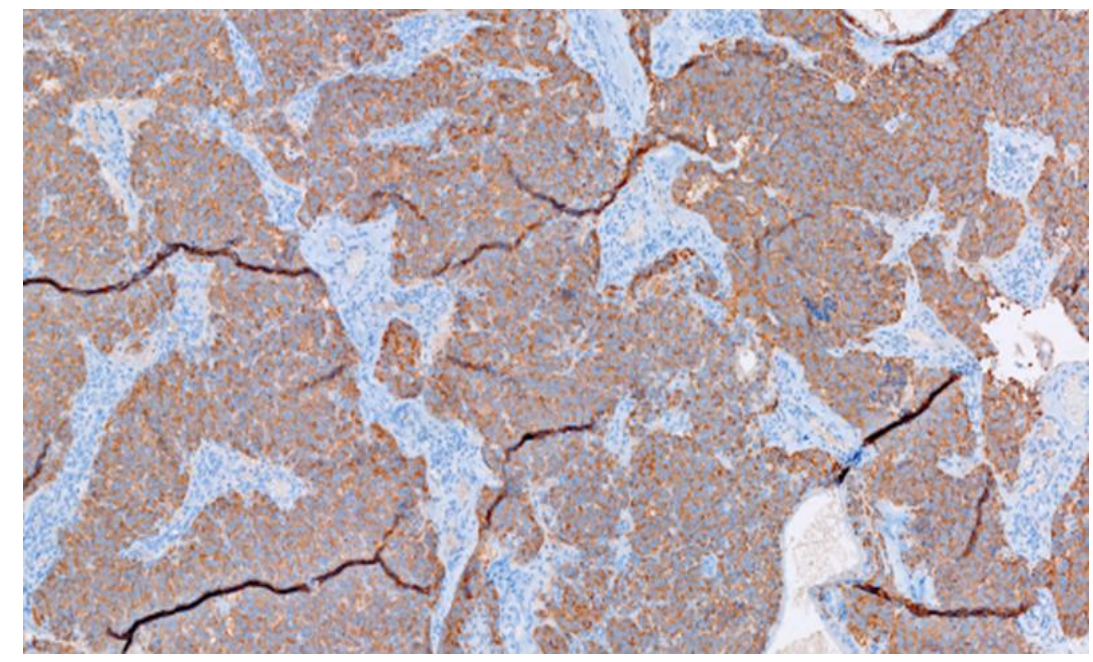

Figure 3 Immunohistochemical slide depicting positivity of synaptophysin marker in the neoplasm 


\section{Discussion}

Olfactory neuroblastoma is an uncommon malignant tumor of the sino-nasal cavity described for the first time in 1924 [9]. ONB arises from olfactory epithelium and it is believed to specifically derive from basal cells based on tumor expression of proteins characteristic of immature olfactory epithelium [10]. In this regard, Carney et al. found that ONB expresses neuroblast marker hASH1 but not OMP, a marker of differentiated olfactory neurons [11].

hASH1 belongs to basic helix-loop-helix family, whom mASH-1 is another member, homologue of hASH1 and frequently expressed in ONB, as evidenced by Ball DW et al [12]. mRNA levels of ASH1 positively correlates with ONB grade and it seems to be related to its neuroendocrine differentiation [10]. hASH1 over expression could act as a fundamental step in ONB carcinogenesis: Osada H et al. showed that suppression of hASH1 expression with RNA interference prompted cell cycle arrest. [13] hASH1 is known to promote ONB progression by inducing anti-apoptotic factor Bcl-2, which positively regulates itself, erythropoietin, erythropoietin receptor, VEGF and HIF-1a. In this manner hASH1 favors tumor progression through neoangiogenesis [14] [15]. Neurotrophins receptors are also known to modulate ONB tumorigenesis and chemotherapeutic resistance. TrkB and p75NR, respectively expressed in 100\% and 60-100\% of ONBs, interact with neurotrophins in a physiologic contest to stimulate growth, differentiation and survival of neuronal cells [16]. In ONB carcinogenesis, TrkB stimulates the maintenance of tumor initiating cells via ERK and Akt pathway activation [10]. Expression of this receptor in pediatric ONBs is associated with poor prognosis and chemotherapeutic resistance [17]. p75NR promotes survival after being properly clivated by alfa and gamma secretases, with consequent proliferation of tumoral clones [18]. TrkA is expressed in $100 \%$ of ONBs; conversely to the previous receptors, it antagonizes tumor progression by acting as a pro-apoptotic and anti-angiogenic factor [17]. Ilan Weinreb et al. found that neurotrophins receptor expression doesn't correlate with Hyam's Grade. This finding supports the theory that ONB grading is not always predictive of prognosis and metastatic potential of ONB, because metastasis is common feature in all grades of ONBs. Consequently, to better understand ONB's behavior is mandatory to assess a combination of markers along with tumor histology [16]. Mao L et al. reported that 70\% of ONBs over-express Patched1, Gli1 and Gli2, suggesting that this way signaling pathway represents another hallmark in ONB carcinogenesis [19]. ONBs show heterogeneous chromosomal aberrations and some of them are predictor of tumor metastatic potential and chemotherapy efficacy. Most ONBs are aneuploid, while the minority of tumors show a diploid or a polyploidy genome [10]. Cytogenetic aberrations predominantly involve chromosomes 2q, 5, 6q, 17, 19, 21q, 22 and 8 [20]. Specifically, gain on 1p chromosome and deletion on chromosome 11 seem to be associated with ONB metastasis [21]. The same evidence fit for tyrosine kinases gene amplification on chromosome $5 q$ and mutation in mTOR/PIK3CA, NF1, CDKN2A and CDKN2C loci [22][23]. Balanced chromosomal translocation and fusion genes are rarely seen in ONBs [10].

\section{Conclusion}

The present manuscript represents a chance to evidence that ONBs have to be considered heterogeneous tumors from a carcinogenetic and clinical course perspective. The complexity of genomic aberrations and gene mutations in ONB are linked to tumorigenesis molecular mechanisms; nevertheless genomic assessment of this kind of tumor could be related as to the clinical course, as its morphological aspects. Then, detection of new genetic hallmarks of ONBs could identify new potential therapeutic targets and predict response rate to standard therapies.

\section{Compliance with ethical standards}

\section{Acknowledgments}

None.

\section{Statement of informed consent}

Informed consent was obtained from the subject of the study."

\section{References}

[1] Diana B. Sinonasal Neuroendocrine Neoplasms: CurrentChallenges and Advances in Diagnosis and Treatment, with a Focus on Olfactory Neuroblastoma, Head NeckPathol. Mar 2018; 12(1): 22-30.

[2] Lester DR. Thompson. Olfactory Neuroblastoma. Head NeckPathol. Sep 2009; 3(3): 252-259. 
[3] Amin MB, Greene FL, Edge SB, et al. The eighthedition AJCC cancerstagingmanual: continuing to build a bridge from a population-based to a more "personalized" approach to cancer staging. CA Cancer J Clin. 2017; 67(2):9399.

[4] Kadish S, Goodman M, Wang CC. Olfactory neuroblastoma. A clinical analysis of 17 cases. Cancer. 1976; 37(3): 1571-1576.

[5] Bell D, Saade R, Roberts D, J Ow T, Kupferman M, DeMonte F, Y Hanna E. Prognostic utility of Hyams histologicalgrading and Kadish-Morita staging systems for esthensioneuroblastoma outcomes. Head Neck Pathol. 2015; 9(1): 51-9.

[6] Saade RE, Hanna EY, Diana B. Prognosis and biology in esthesioneuroblastoma: the emerging role of Hyams grading system. Curr Oncol Rep. 2015; 17(1):423.

[7] Ow TJ, Bell D, Kupferman ME, Demonte F, Hanna EY. Esthesioneuroblastoma. Neurosurg Clin N Am. 2013; 24(1):51-65.

[8] Lee DC, Bpnny C, Jason KR, Manuel F Jr, Jay JL. Chemotherapy in Esthensioneuroblastoma/Olfactory Neuroblastoma: An Analysis of the SurveillanceEpidemiology and End Results (SEER) 1973-2015 Database. Am J ClinOncol. Mar 2020; 43(3): 203-209.

[9] Barnes L, Eveson JW, Reichart P, Sidransky D. Tumours of the nasal cavity and paranasal sinuses. Pathol Genet Head Neck Tumours. 2005.

[10] \Piotr C, Michal K, Johannes H. Genetic and molecular alterations in olfactory neuroblastoma: implications for pathogenesis, prognosis and treatment. Oncotarget. 9 Aug 2016; 7(32): 53584- 52596.

[11] Jiang SX, Kameya T, Asamura H, Umezawa A, Sato Y, Shinada J, Kawakubo Y, Igarashi T, Nagai K, Okayasu I. hASH1 expression is closely correlated with endocrine phenotype and differentiation extent in pulmonary neuroendocrine tumors. Mod Pathol. 2004; 17:222-229.

[12] Ball DW, Azzoli CG, Baylin SB, et al. Identification of a human achaete-scute homolog highly expressed in neuroendocrine tumors. Proc Natl Acad Sci USA. 1993; 90(12): 5648-5652.

[13] Osada H, Tatematsu Y, Yatabe Y, Horio Y, Takahashi T. ASH1 gene is a specifictherapeutic target for lung cancers with neuroendocrine features. Cancer Res. 2005; 65: 10680-10685.

[14] Diensthuber M, Potinius M, Rodt T, Stan AC, Welkoborsky H-J, Samii M, Schreyögg J, Lenarz T, Stöver T. Expression of bcl-2 is associated with microvessel density in olfactory neuroblastoma.J Neuro oncol. 2008; 89: 131-139.

[15] Hoff PM, Machado KK. Role of angiogenesis in the pathogenesis of cancer. Cancer Treat Rev. 2012; 38: 825-833.

[16] Weinreb I, Goldstein D, Irish J, Perez-Ordonez B. Expression patterns of Trk-A, Trk-B, GRP78, and p75NRT in olfactory neuroblastoma. Hum Pathol. 2009; 40: 1330-1335.

[17] Minturn JE, Evans AE, Villablanca JG, Yanik GA, Park JR, Shusterman S, Groshen S, Hellriegel ET, Bensen-Kennedy D, Matthay KK, Brodeur GM, Maris JM. Phase I trial of lestaurtinib for children with refractory neuroblastoma: a new approaches to neuroblastoma therapy consortium study. Cancer Chemother Pharmacol. 2011; 68: 10571065.

[18] Forsyth PA, Krishna N, Lawn S, Valadez JG, Qu X, Fenstermacher DA, Fournier M, Potthast L, Chinnaiyan P, Gibney GT, Zeinieh M, Barker PA, et al. p75 neurotrophin receptor cleavage by $\alpha$ - and $\gamma$-secretases is required for neurotrophin-mediated proliferation of brain tumor-initiating cells. J Biol Chem. 2014; 289: 8067-8085.

[19] Mao L, Xia YP, Zhou YN, et al. Activation of sonic hedgehog signaling pathway in olfactory neuroblastoma. Oncology. 2009; 77(3-4): 231-243.

[20] Holland H, Koschny R, Krupp W, et al. Comprehensive cytogenetic characterization of an esthesioneuroblastoma. Cancer Genet Cytogenet. 2007; 173(2): 89-96.

[21] Bockmuhl U, You X, Pacyna-Gengelbach M, Arps H, Draf W, Petersen I. CGH pattern of esthesioneuroblastoma and their metastases. Brain Pathol. 2004; 14(2): 158-163.

[22] Czapiewski P, Kunc M, Haybaeck J. Genetic and molecular alterations in olfactory neuroblastoma: implications for pathogenesis, prognosis and treatment. Oncotarget. 2016; 7(32): 52584-52596.

[23] de la LazoVega L, McHugh JB, Cani AK, et al. Comprehensive molecular profiling of olfactory neuroblastoma identifies potentially targetable FGFR3 amplifications. Mol Cancer Res. 2017; 15(11): 1551-1557. 